\title{
Suppression of star formation in early-type galaxies by feedback from supermassive black holes
}

Kevin Schawinski ${ }^{1}$, Sadegh Khochfar ${ }^{1}$, Sugata Kaviraj ${ }^{1}$, Sukyoung K. Yi ${ }^{2}$, Alessandro Boselli ${ }^{3}$, Tom Barlow ${ }^{4}$, Tim Conrow ${ }^{4}$, Karl Forster ${ }^{4}$, Peter G. Friedman ${ }^{4}$, D. Chris Martin ${ }^{4}$, Patrick Morrissey ${ }^{4}$, Susan Neff ${ }^{5}$, David Schiminovich ${ }^{6}$, Mark Seibert ${ }^{4}$, Todd Small ${ }^{4}$, Ted K. Wyder ${ }^{4}$, Luciana Bianchi ${ }^{7}$, Jose Donas ${ }^{3}$, Tim Heckman ${ }^{7}$, Young-Wook Lee ${ }^{2}$, Barry Madore ${ }^{8}$, Bruno Milliard ${ }^{3}$, R. Michael Rich $^{9} \&$ Alex Szalay ${ }^{7}$

${ }^{1}$ Department of Physics, University of Oxford, Oxford OX1 3RH, UK

${ }^{2}$ Center for Space Astrophysics, Yonsei University, Seoul 120-749, Korea

${ }^{3}$ Laboratoire d'Astrophysique de Marseille, 13376 Marseille Cedex 12, France

${ }^{4}$ California Institute of Technology, MC 405-47, Pasadena, CA 91125

${ }^{5}$ Laboratory for Astronomy and Solar Physics, NASA Goddard Space Flight Center, Greenbelt, MD 20771

${ }^{6}$ Department of Astronomy, Columbia University, MC 5246, New York, NY 10027

${ }^{7}$ Department of Physics and Astronomy, Johns Hopkins University, Baltimore, MD 21218

${ }^{8}$ Observatories of the Carnegie Institution of Washington, 813 Santa Barbara St., Pasadena, CA 91101

${ }^{9}$ Department of Physics and Astronomy, UCLA, Los Angeles, CA 90095-1562

Detailed high resolution observations of the innermost central parts of nearby galaxies have revealed the presence of supermassive black holes ${ }^{1-4}$. These black holes may interact with 
their host galaxies by means of 'feedback' in the form of energy and material jets; this feedback affects the evolution of the host and gives rise to the observed relations between the black hole and the host ${ }^{5}$. Here we report observations of the ultraviolet emissions of massive early-type galaxies. We derive an empirical relation for a critical black-hole mass (as a function of velocity dispersion) above which the outflows from these black holes suppress star formation in their hosts by heating and expelling all available cold gas. Supermassive black holes are negligible in mass compared to their hosts but nevertheless seem to play a critical role in the star formation history of galaxies.

The near-UV (NUV) detector of the Galaxy Evolution Explorer satellite GALEX ${ }^{6}$ covers a range in wavelength between 1771 and $2831 \AA$ and is extremely sensitive to young stellar populations. With it, we can detect small mass fractions of $1-3 \%$ of young stars formed within the last billion years ${ }^{7,8}$. This high sensitivity allows us to trace ongoing residual star formation in present day early-type galaxies (ETGs). We select a volume-limited sample of visually inspected ETGs from the Sloan Digital Sky Survey ${ }^{9}$ that have been observed by GALEX. We remove active galactic nucleus (AGN) candidates from our sample to avoid confusion between the observed UV emissions from the AGN and from young stars ${ }^{1}$.

We use $N U V-r$ colour as a probe of small amounts of recent star formation (RSF) and find that the fraction of ETGs that show signs of RSF is strongly correlated with the stellar velocity dispersion $\sigma$, (hereafter "RSF- $\sigma$ relation"), but not with the luminosity of the galaxy (see Figure 1). The correlation with $\sigma$ cannot be explained by a systematic trend of $\sigma$ with internal extinction, 
because such a trend would be exact opposite ${ }^{11}$ to that observed.

We use a semi-analytic model of galaxy evolution in the $\Lambda$ CDM paradigm to interpret these results $^{2,3,14}$ (for model details see Supplements). Our simulation includes all the systematic processes that affect galaxy evolution and takes into account the different merger and star formation histories of galaxies ${ }^{15}$. When we run this simulation and investigate the predicted amount of recent star formation in low redshift ETGs, we find that all galaxies underwent enough star formation within the last billion years to be detected by the extremely sensitive GALEX NUV detector. Massive galaxies continued to gain cold gas as fuel for star formation from mergers with satellites and through the cooling of hot gas present in those galaxies.

This overproduction of stars in massive galaxies is a known problem in models of galaxy evolution. In order to prevent star formation, a shutdown mechanism is required: cold gas must be heated up and expelled to prevent it from turning into stars. Feedback from supernovae ( $\mathrm{SNe}$ ) was once a prime suspect, but it turned out to be too low to be effective in massive galaxies which have deep potential wells ${ }^{16}$. All the systematics implicit in the $\Lambda$ CDM paradigm, including feedback from SNe have already been included in our simulation, so we turn to feedback from active galactic nuclei (AGN) powered by supermassive black holes (SMBH) as the remaining most likely mechanism sufficiently powerful to shut down star formation in massive galaxies to reproduce the results of our observations ${ }^{17-19}$.

Energetic outflows caused by gas falling onto the supermassive black holes thought to reside at the centres of galaxies can self-regulate the growth of the black hole and the star formation in 
the galaxy by heating the available gas and blowing it out ${ }^{5}$. Numerical simulations have shown that the energy input from a black hole in such an AGN phase is capable of doing this ${ }^{20}$. As $\mathrm{SNe}$ feedback is incapable of producing the quiescent galaxies we observe with GALEX, we conclude that AGN feedback is the most likely process by which the available gas is heated and expelled in massive ETGs.

In addition to this, the nature of the observed correlation with sigma (see Figure 1) allows us to infer more about the way in which AGN feedback regulates galaxy evolution. The masses of black holes correlate strongly with various properties of the host galaxies and the relation thought to have the smallest intrinsic scatter is that between $M_{\bullet}$ and the velocity dispersion $\sigma$ as a proxy for the depth of the gravitational potential ${ }^{1,3,4}$. By considering the maximum strength of an active galactic nucleus given in terms of the Eddington luminosity $L_{e d d} \propto M_{\bullet}$ and expressing the gravitational potential depth by the stellar velocity dispersion $\sigma$, it is possible to derive a minimum black hole mass for which the outflow is capable of depleting all gas from the potential ${ }^{5}$. According to our observations, massive ETGs which have large values of $\sigma$, are on average less likely to have undergone recent star formation compared to less massive ETGs: the "RSF- $\sigma$ relation". Thus, for a given velocity dispersion, we assume that there is a critical supermassive black hole mass above which the feedback powered by the black hole is sufficiently powerful to heat up and/or expel all gas in the host galaxy, thus preventing it from forming any stars even at the $1 \%$ level.

The observed intrinsic scatter in the $M_{\bullet}-\sigma$ relation gives a range of black hole masses for a given value of $\sigma$. Hence, in order to reproduce the decreasing fraction of RSF ETGs with increasing 
$\sigma$, the $M_{\bullet, c}-\sigma$ relation should be less steep than the $M_{\bullet}-\sigma$ relation itself. We can derive an empirical $M_{\bullet, c}-\sigma$ relation from our observed trend assuming a slope and scatter for the $M_{\bullet}-\sigma$ relation. The $M_{\bullet}-\sigma$ relation is usually expressed as a power law of the form $M_{\bullet}=a_{0}(\sigma / 200)^{a_{1}}$, so we also try to find such parameters $a_{0}$ and $a_{1}$ in the new $M_{\bullet, c}-\sigma$ relation that result in the observed "RSF- $\sigma$ relation". We generate black hole masses for the galaxies in our sample by putting their velocity dispersions into the $M_{\bullet}-\sigma$ relation given by Tremaine et al. ${ }^{3}$ and perturbing the black hole masses by the scatter of the relation; we do this ten times for each galaxy (Figure 2 (a)). Then we vary $a_{0}$ and $a_{1}$ until we find the best match to the observed RSF- $\sigma$ relation. We find that with $95 \%$ confidence $M_{\bullet, c}=\left(1.05_{-0.12}^{+0.07}\right) \times 10^{8}\left(\frac{\sigma}{200}\right)^{3.2_{-0.6}^{+0.5}}$. As we are studying a volume and magnitude-limited sample, the $M_{\bullet, c}-\sigma$ relation derived from the Monte-Carlo simulation is only an approximation and we need to perform full simulations, as they are complete in $\sigma$ as well as in absolute magnitude, to derive the actual $M_{\bullet, c}-\sigma$ relation.

We perform a more realisitc simulation using our semi-analytic models of galaxy evolution in order to test the plausibility of the hypothesis of a critical black hole mass controlling star formation via AGN feedback. This is a standard semi-analytic simulation in the $\Lambda C D M$ paradigm that already includes various heating and cooling processes (e.g., SNe). Our model does indeed reproduce the observed trend, but only when AGN feedback is included, and the best agreement with the observations (Figure 1) is achieved by the relation $M_{\bullet, c}=1.26 \times 10^{8}\left(\frac{\sigma}{200}\right)^{3.65}$ (Figure 2b), which is in good agreement with our initial guess. The agreement in the lowest $\sigma$-bin is poorer than in the others due to an increased scatter in our simulated Faber-Jackson relation relative to the observed one $^{21}$. The supermassive black holes grown in our simulation have an intrinsic scatter of 
a factor of $1.5 \mathrm{in}$ mass and are in good agreement with the relation suggested by Tremaine et al. ${ }^{3}$

As our $M_{\bullet, c}-\sigma$ relation is less steep than the $M_{\bullet}-\sigma$ relation we expect an increasing fraction of star forming early-type galaxies with decreasing velocity dispersion until eventually no AGN will be sufficiently powerful to shut down all star formation. Boselli et al. $^{4}$ find that the $N U V-V$ (optical) and $N U V-H$ (near IR) colour-magnitude relations for the early-type population of the Virgo cluster shows precisely this kind of trend: low luminosity - and therefore less massive Virgo early-type galaxies are increasingly bluer and eventually all lie below the RSF cut-off. We use stellar models ${ }^{23}$ to convert our RSF criterion to the $N U V-V$ colours of the Virgo galaxies and find that below $\sigma \sim 80 \mathrm{kms}^{-1}$ all early-types are classified as RSF. Our simulations agree with this cutoff very well.

The possible implications of the $M_{\bullet, c}-\sigma$ relation on galaxy formation and the growth of black holes can be summarized by three main regions (see Figure 3): First, the low $\sigma$-region, in which the majority of black holes are too small to be able to halt star formation in their host galaxies by feedback during their initial accretion phase. During this phase, black holes grow mainly by accreting gas at the Eddington rate and are only limited in their growth by the amount of available fuel. Star formation on the other hand is regulated by the energy output from supernovae into the interstellar medium and will be proportional to the surface density of the gas ${ }^{24}$. This region extends up to $\sigma \sim 80 \mathrm{~km} \mathrm{~s}^{-1}$ as suggested earlier. The next phase is at $80 \mathrm{kms}^{-1}<\sigma<240 \mathrm{kms}^{-1}$. Black holes are now becoming on average massive enough to be able to terminate star formation by their feedback and at the same time limit their available fuel. The mass growth of the black holes is still 
dominated by accretion but mergers between black holes start playing an increasingly important role. Most host galaxies will be identified as AGN or QSO when observed during the accretion phase onto the black hole. We sub-divide this region into two: at the lower end, a minority of galaxies will have critical black holes; the black hole still grow mostly via accretion. At the high end, most black holes are critical and so most of the mass growth now occurs via mergers. The boundary between these two is at the intersection between the $M_{\bullet}-\sigma$ and the critical relation, i.e. $\sigma \sim 165 \mathrm{kms}^{-1}$. Finally, for galaxies with $\sigma>240 \mathrm{kms}^{-1}$ the vast majority of black holes is massive enough to halt all star formation and so star formation becomes virtually prohibited in such massive galaxies. Both the galaxy and the supermassive black holes now mainly grow via dry mergers ${ }^{25}$. Further observations of nearby galaxies hosting supermassive black holes in combination with star formation estimates will help to shed more light into the scenario we propose and will place a strong test for the existence of "a critical black hole mass" in galaxies.

1. Gebhardt, K. et al. A Relationship between Nuclear Black Hole Mass and Galaxy Velocity Dispersion. Astrophys. J. Lett. 539, L13-L16 (2000).

2. Merritt, D. \& Ferrarese, L. The $M_{b h^{-}} \sigma$ Relation for Supermassive Black Holes. Astrophys. $J$ 547, 140-145 (2001).

3. Tremaine, S. et al. The Slope of the Black Hole Mass versus Velocity Dispersion Correlation. Astrophys. J. 574, 740-753 (2002).

4. Ferrarese, L. \& Ford, H. Supermassive Black Holes in Galactic Nuclei: Past, Present and Future Research. Space Science Reviews 116, 523-624 (2005). 
5. Silk, J. \& Rees, M. J. Quasars and galaxy formation. Astron. Astrophys. 331, L1-L4 (1998).

6. Morrissey, P. et al. The On-Orbit Performance of the Galaxy Evolution Explorer. ApJL 619, L7-L10 (2005).

7. Yi, S. K. et al. Galaxy Evolution Explorer Ultraviolet Color-Magnitude Relations and Evidence of Recent Star Formation in Early-Type Galaxies. ApJL 619, L111-L114 (2005).

8. Kaviraj, S. et al. UV-optical colours as probes of early-type galaxy evolution. ArXiv Astrophysics e-prints (2006). arXiv:astro-ph/0601029.

9. York, D. G. et al. The Sloan Digital Sky Survey: Technical Summary. Astron. J. 120, 15791587 (2000).

10. Schawinski, K. et al. The effect of environment on the UV colour-magnitude relation of earlytype galaxies. ArXiv Astrophysics e-prints (2006). arXiv:astro-ph/0601036.

11. Lauer, T. R. et al. The Centers of Early-Type Galaxies with Hubble Space Telescope. V. New WFPC2 Photometry. AJ 129, 2138-2185 (2005).

12. White, S. D. M. \& Frenk, C. S. Galaxy formation through hierarchical clustering. Astrophys. J. 379, 52-79 (1991).

13. Springel, V., White, S. D. M., Tormen, G. \& Kauffmann, G. Populating a cluster of galaxies I. Results at [formmu2]z=0. Mon. Not. R. Astron. Soc. 328, 726-750 (2001).

14. Khochfar, S. \& Burkert, A. On the origin of isophotal shapes in elliptical galaxies. Mon. Not. R. Astron. Soc. 359, 1379-1385 (2005). 
15. Khochfar, S. \& Silk, J. On the Origin of Stars in Bulges and Elliptical Galaxies. ArXiv Astrophysics e-prints (2005). arXiv:astro-ph/0509375.

16. Dekel, A. \& Silk, J. The origin of dwarf galaxies, cold dark matter, and biased galaxy formation. Astrophys. J 303, 39-55 (1986).

17. Di Matteo, T., Springel, V. \& Hernquist, L. Energy input from quasars regulates the growth and activity of black holes and their host galaxies. Nature 433, 604-607 (2005).

18. Croton, D. J. et al. The many lives of active galactic nuclei: cooling flows, black holes and the luminosities and colours of galaxies. Mon. Not. R. Astron. Soc. 365, 11-28 (2006).

19. Hopkins, P. F. et al. Black Holes in Galaxy Mergers: Evolution of Quasars. Astrophys. J. 630, 705-715 (2005).

20. Springel, V., Di Matteo, T. \& Hernquist, L. Modelling feedback from stars and black holes in galaxy mergers. Mon. Not. R. Astron. Soc. 361, 776-794 (2005).

21. Bernardi, M. et al. Early-Type Galaxies in the Sloan Digital Sky Survey. III. The Fundamental Plane. Astron. J. 125, 1866-1881 (2003).

22. Boselli, A. et al. UV Properties of Early-Type Galaxies in the Virgo Cluster. Astrophys. J. Lett. 629, L29-L32 (2005).

23. Yi, S. K. Uncertainties of Synthetic Integrated Colors as Age Indicators. Astrophys. J. 582, 202-214 (2003). 
24. Kennicutt, R. C. The Global Schmidt Law in Star-forming Galaxies. Astrophys. J. 498, 541-+ (1998).

25. Khochfar, S. \& Burkert, A. The Importance of Spheroidal and Mixed Mergers for Early-Type Galaxy Formation. Astrophys. J. Lett. 597, L117-L120 (2003).

Acknowledgements We would like to thank John Magorrian for discussions and comments. GALEX (Galaxy Evolution Explorer) is a NASA Small Explorer, launched in April 2003. We gratefully acknowledge NASA's support for construction, operation, and science analysis for the GALEX mission, developed in cooperation with the Centre National d'Etudes Spatiales of France and the Korean Ministry of Science and Technology. This work was supported by grant No. R01-2006-000-10716-0 from the Basic Research Program of the Korea Science \& Engineering Foundation to S.K.Y.

Competing Interests The authors declare that they have no competing financial interests.

Auhtor Information K.S., Sadegh Khochfar, Sugata Kaviraj, S.K.Y. have performed the data sampling, analysis, interpretation, model construction, and writing of the manuscript. A.B. supplied the Virgo galaxy data, and the rest of the authors contributed to the data acquisition using the GALEX satellite. Correspondence and requests for materials should be addressed to S.K.Y. (email: yi@yonsei.ac.kr). 


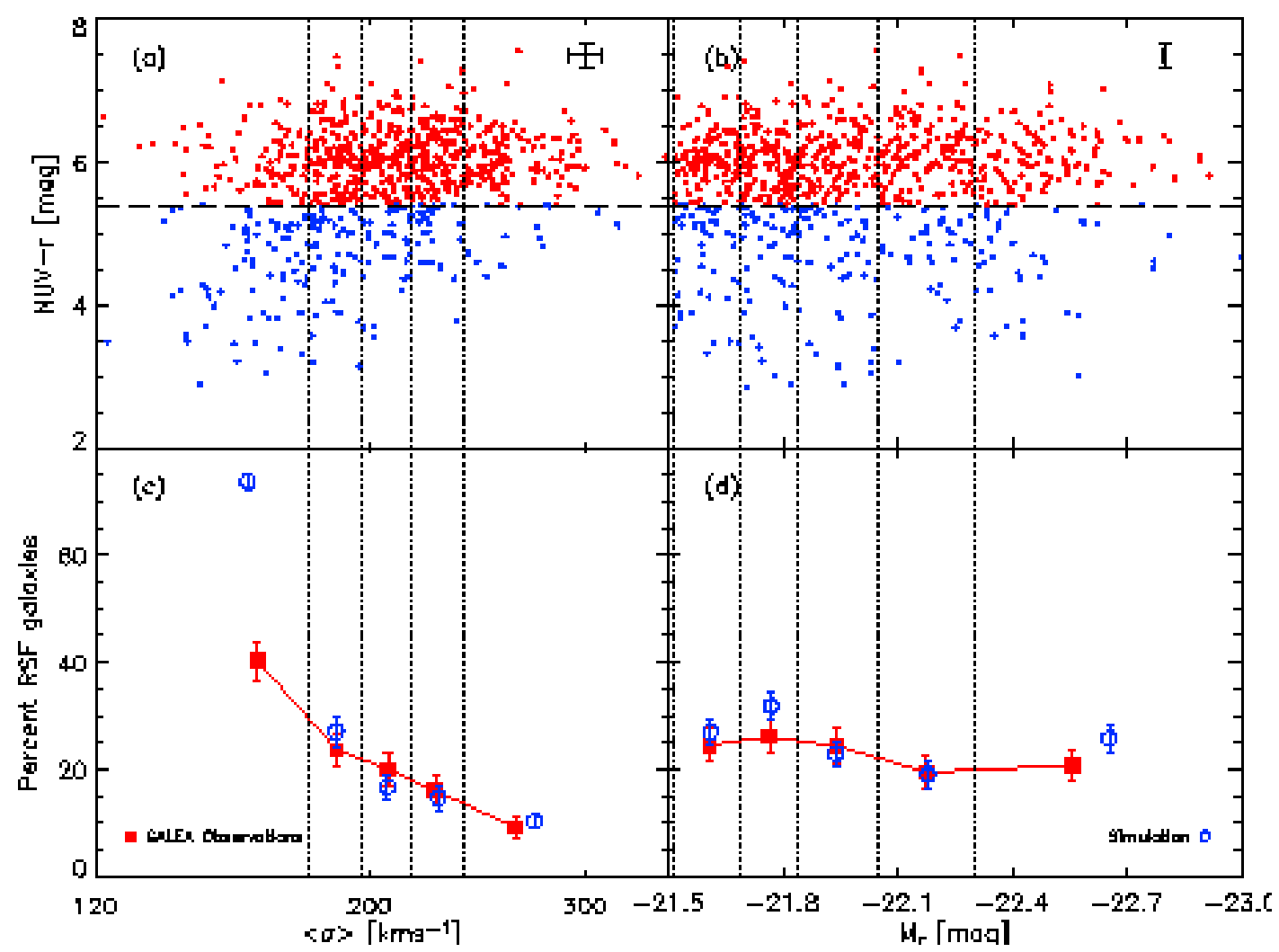

Figure 1: The relationship between stellar velocity dispersion, luminosity and the fraction of star-forming early-type galaxies In panels (a) and (b), we show the observed UV colour-sigma and colour-magnitude relations. In the case of the colour-sigma relation, the fraction of blue earlytype galaxies declines sharply as a function of sigma. In panels (c) and (d), we show the derived fractions of RSF (recent star formation) galaxies as a function of velocity dispersion and absolute magnitude. In red, we show the observed fractions, which include those early-type galaxies so faint in the UV that they are not detected by the GALEX NUV detector. The blue points are the fractions of those galaxies in the model that correspond to having had between 1 and $3 \%$ of stellar mass growth within the last 1 billion years. Error bars show Poisson errors. The vertical lines delineate the equal-number $\sigma$ bins. 


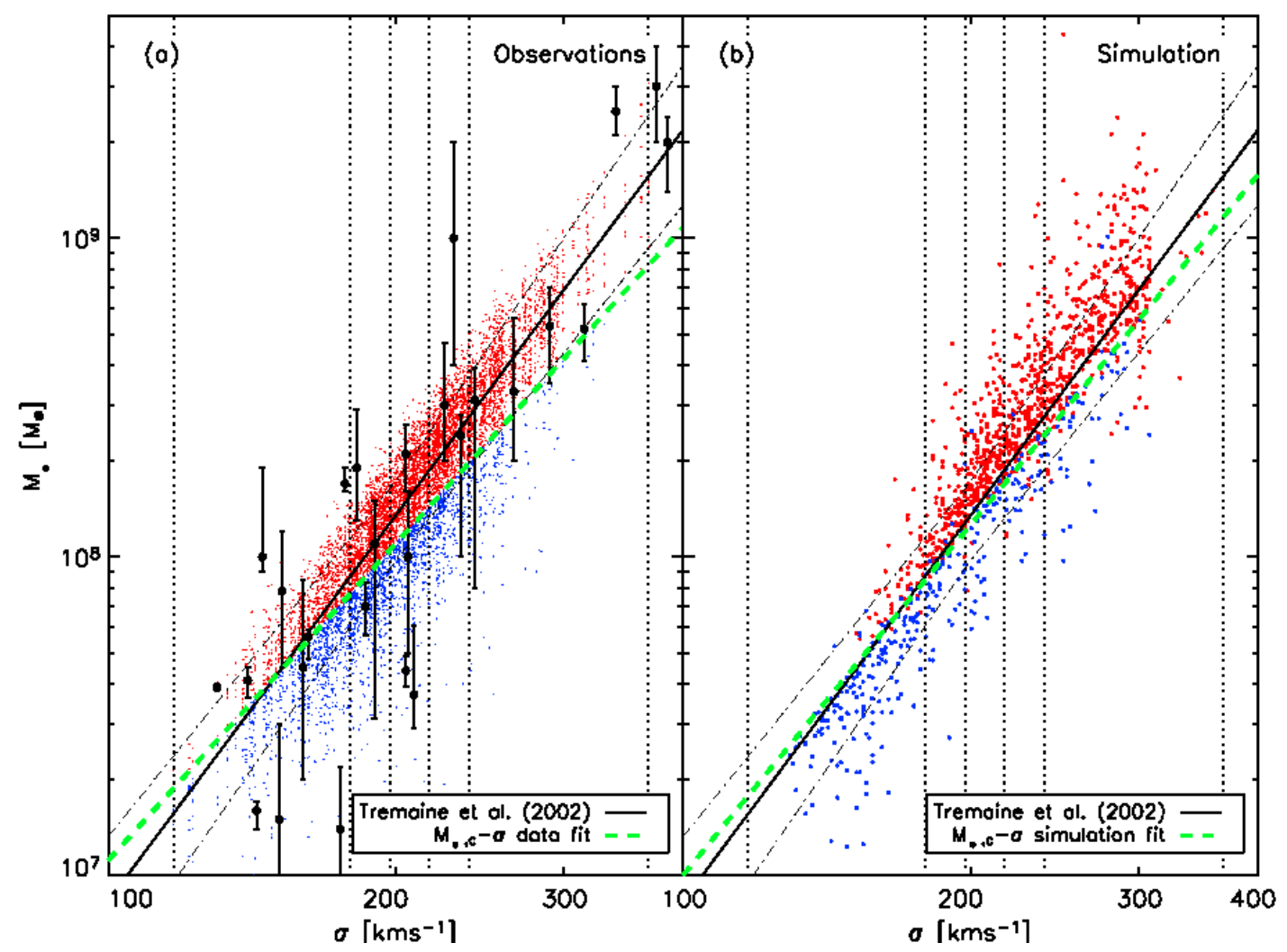

Figure 2: The $M_{\bullet}-\sigma$ and the critical supermassive black hole mass- $\sigma$ relation (a) The observed $M_{\bullet}-\sigma$ data points (black dots) with their errors taken from Tremaine et al. ${ }^{3}$ The solid line is the best fit to the $M_{\bullet}-\sigma$ relation, with the dash-dotted line showing the 1- $\sigma$ scatter. Based on this relation we perform a Monte-Carlo simulation for our galaxies (small dots). We colour these simulated galaxies depending on whether they lie above or below the critical relation in red (quiescent) and blue (RSF) that we derived by fitting the RSF fraction to the observed values (Figure 1). The vertical lines delineate the equal-number $\sigma$ bins of the data. (b) We show our semi-analytic galaxy models adopting $M_{\bullet, c}-\sigma$ relation shown in Figure 2(b), in comparison to the empirical $M_{\bullet}-\sigma$ relation. Red and blue dots are the model galaxies that did and did not have recent star formation in the last billion years. A new demarcation slope that effectively separates these galaxies has a $\sigma^{3.65}$ dependence (dashed green) which is close to the Monte-Carlo one in (a). 


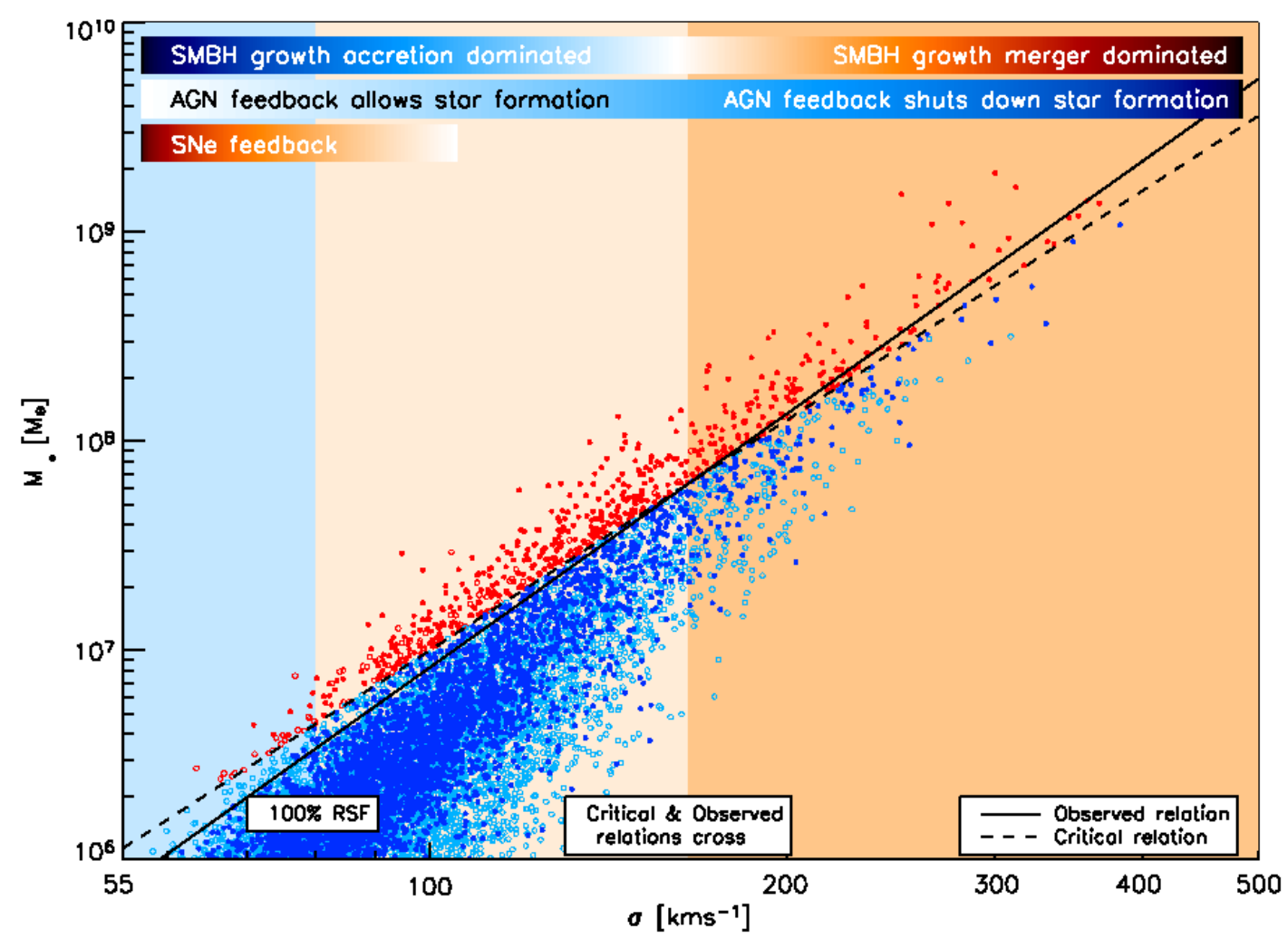

Figure 3: A schematic view of how the critical supermassive black hole mass - velocity dispersion relation regulates galaxy evolution The black hole masses and velocity dispersions of our simulated galaxies down to $\sigma \sim 50 \mathrm{kms}^{-1}$. The full points are early-type galaxies, while empty circles are late-type galaxies. Red and blue symbols denote quiescent and RSF galaxies, respectively. We show the observed $M_{\bullet}-\sigma$ relation (solid line) together with the best SAM critical relation (dashed). We indicate the various regimes by colour. 


\section{Supplements}

\section{Galaxy Formation Modelling}

The main strategy behind the modelling approach we follow is to first calculate the collapse and merging history of individual dark matter halos, which is governed purely by gravitational interactions, and secondly to calculate the more complex physics of the baryons inside these dark matter halos, including e.g. radiative cooling of the gas, star formation and feedback from supernovae by simplified prescriptions on top of the dark matter evolution. Each of the dark matter halos consist of three main components which are distributed among individual galaxies inside them. A stellar, cold and hot gas component, where the latter is only attributed to central galaxies, the most massive galaxies inside individual halos. In the following sections, we will describe briefly the recipes used to calculate these different components which are mainly based on recipes presented in Kauffmann et al..$^{5}$ (hereafter, K99) and Springel et al. ${ }^{3}$ (hereafter, S01), and we refer readers for more details on model implementations to their work and references therein.

Throughout this paper we use the following set of cosmological parameters: $\Omega_{0}=0.3$, $\Omega_{\Lambda}=0.7, \Omega_{b} / \Omega_{0}=0.15, \sigma_{8}=0.9$ and $h=0.65$.

Dark Matter Evolution We calculate the merging history of dark matter halos according to the prescription presented in Somerville et al. ${ }^{6}$. This approach has been shown to produce merging histories and progenitor distributions in reasonable agreement with result from N-body simulations of cold dark matter structure formation in a cosmological context ${ }^{7}$. The merging history of dark 
matter halos is reconstructed by breaking each halo up into progenitors above a limiting minimum progenitor mass $M_{\min }$. This mass cut needs to be chosen carefully as it ensures that the right galaxy population and merging histories are produced within the model. Progenitor halos with masses below $M_{\min }$ are declared as accretion events and their histories are not followed further back in time. Progenitors labelled as accretion events should ideally not host any significant galaxies in them and be composed mainly of primordial hot gas at the progenitor halo's virial temperature. To achieve a good compromise between accuracy and computational time, we use $M_{\min }=10^{10} M_{\odot}$, which ensures that the results presented here are unaffected by numerical resolution effects.

Baryonic Physics As mentioned above, once the merging history of the dark matter component has been calculated, it is possible to follow the evolution of the baryonic content in these halos forward in time. We assume each halo consists of three components: hot gas, cold gas and stars, where the latter two components can be distributed among individual galaxies inside a single dark matter halo. The stellar components of each galaxy are further divided into bulge and disc, to allow morphological classifications of model galaxies. In the following, we describe how the evolution of each component is calculated.

Gas Cooling \& Reionisation Each branch of the merger tree starts at a progenitor mass of $M_{\min }$ and ends at a redshift of $z=0$. Initially, each halo is occupied by hot primordial gas which was captured in the potential well of the halo and shock-heated to its virial temperature $T_{v i r}=$ $35.9\left[V_{c} /\left(\mathrm{km} \mathrm{s}^{-1}\right)\right]^{2} \mathrm{~K}$, where $V_{c}$ is the circular velocity of the halo ${ }^{2,5}$. Subsequently this hot gas component is allowed to radiatively cool and settles down into a rotationally supported gas disc at 
the centre of the halo, which we identify as the central galaxy $y^{2,8,9}$. The rate at which hot gas cools down is estimated by calculating the cooling radius inside the halo using the cooling functions provided by ${ }^{10}$ and the prescription in S01. In the case of a merger between halos we assume that all of the hot gas present in the progenitors gets shock-heated to the virial temperature of the remnant halo, and this gas can cool down only onto the new central galaxy which is the central galaxy of the most massive progenitor halo. The central galaxy of the less massive halo will become a satellite galaxy orbiting inside the remnant halo. In this way, a halo can host multiple satellite galaxies, depending on the merging history of the halo, but will always only host one central galaxy onto which gas can cool. The cold gas content in satellite galaxies is given by the amount present when they first became satellite galaxies and does not increase, but decreases due to ongoing star formation and supernova feedback.

In the simplified picture adopted above, the amount of gas available to cool down is limited only by the universal baryon fraction $\Omega_{b} h^{2}=0.0224^{11}$. However, in the presence of a photoionising background the fraction of baryons captured in halos is reduced ${ }^{12,13}$ and we use the recipe of Somerville et al. ${ }^{14}$, which is based on a fitting formulae derived from hydrodynamical simulations $^{13}$, to estimate the amount of baryons in each halo. For the epoch of reionisation, we assume $z_{\text {reion }}=20$, which is in agreement with observations of the temperature-polarisation correlation of the cosmic microwave background ${ }^{15}$.

Star Formation in Discs and Supernova Feedback Once cooled gas has settled down in a disc, we allow for fragmentation and subsequent star formation according to a parameterised global 
Schmidt-Kennicutt law ${ }^{16}$ of the form $\dot{M}_{*}=\alpha M_{\text {cold }} / t_{d y n, g a l}$, where $\alpha$ is a free parameter describing the efficiency of the conversion of cold gas into stars, and $t_{d y n, g a l}$ is assumed to be the dynamical time of the galaxy and is approximated to be 0.1 times the dynamical time of the dark matter halo ${ }^{5}$. As in K99 we allow star formation only in halos of $V_{c}<350 \mathrm{~km} / \mathrm{s}$ to avoid excessively-massive central galaxies in clusters.

Feedback from supernovae plays an important role in regulating star formation in small mass halos and in preventing excessively-massive satellite galaxies from forming. We implement feedback based on the prescription presented in K99 with

$$
\Delta M_{\text {reheat }}=\frac{4}{3} \epsilon \frac{\eta_{S N} E_{S N}}{V_{c}^{2}} \Delta M_{*} .
$$

Here we introduce a second free parameter $\epsilon$ which represents our ignorance about the efficiency with which the energy from supernovae reheats the cold gas. The expected number of supernovae per solar mass of stars formed is given by $\eta_{S N}=5 \times 10^{-3}$, taken as the value for the Scalo initial mass function ${ }^{17}$, and $E_{S N}=10^{51} \mathrm{erg}$ is the energy output from each supernova. We take $V_{c}$ as the circular velocity of the halo in which the galaxy was last present as a central galaxy.

Galaxy Mergers We allow for mergers between galaxies residing in a single halo. As mentioned earlier, each halo is occupied by one central galaxy and a number of satellite galaxies depending on the past merging history of the halo. Whenever two halos merge, the galaxies inside them are going to merge on a time-scale which we calculate by estimating the time it would take the satellite to reach the centre of the halo under the effects of dynamical friction. Satellites are assumed to merge only with central galaxies and we set up their orbits in the halo according to the prescription 
of K99, modified to use the Coulomb logarithm approximation of S01.

If the mass ratio between the two merging galaxies is $M_{g a l, 1} / M_{g a l, 2} \leq 3.5\left(M_{g a l, 1} \geq M_{g a l, 2}\right)$ we declare the event as a major merger and the remnant will be an elliptical galaxy and the stellar components and the gas will be treated according to the prescriptions below. In the case of minor merger $M_{g a l, 1} / M_{g a l, 2}>3.5$ the cold gas in the disc of the smaller progenitor is assumed to settle down in the gas disc of the remnant and its stars contribute to the bulge component of the remnant ${ }^{5}$.

Formation of Ellipticals and Bulges It is suggested that major mergers will lead to the formation of elliptical galaxies ${ }^{18}$. Indeed detailed numerical simulations in the last decade seem to support this hypothesis ${ }^{19,20}$, and we wil assume in the following that major mergers disrupt the discs in progenitor galaxies as seen in various numerical simulations and relax to a spheroidal distribution. During the merger, any cold gas in the discs of the progenitor galaxies is assumed to be funnelled into the centre of the remnant where it ignites a starburst which transforms all of the cold gas into stars contributing to the spheroidal component ${ }^{3,5}$. The second assumption is certainly a simplification of what might happen since we neglect the possibility that not all of the cold gas is funnelled to the centre but some fraction of it may for example settle down onto an extended disk which continues to grow inside-out by fresh supply of gas from tidal tails ${ }^{21-24}$. The results of Barnes ${ }^{24}$ indicate that $40 \%-80 \%$ of the initial gas in the disc could end up in the central region of the remnant and be consumed in a starburst. The exact amount is somewhat dependent on the merger geometry and on the mass ratio of the merger. Unfortunately, a sufficiently large survey investigating the gas inflow to the centres of merger remnants is not available yet, so we use the simplified approach of 
assuming that all cold gas gets used up in the central starburst. This prescription for the fate of the cold gas results in an overestimate of the spheroid masses and an underestimate of the secondary disc components in our model. This is not very significant for massive ellipticals since they are mainly formed in relatively non-dissipative mergers ${ }^{25}$.

Formation and Growth of Super-Massive Black Holes We here follow the model introduced in Kauffmann \& Haehnelt ${ }^{26}$ in which super-massive black holes get formed and fed during major mergers of glaxies. The assumption is to say that a fraction of the the cold gas available in the progenitor discs, that is funnelled into the centre of the remnant, will be accreted onto the black hole. Kaufmann \& Haehnelt introduced the following scaling law for the effectivness of this procces:

$$
\dot{M}_{\bullet}=\frac{f_{b h} M_{c o l d}}{1+\left(280 \mathrm{~km} \mathrm{~s}^{-1} / V_{c}\right)^{2}},
$$

where $M_{\text {cold }}$ is the amount of cold gas available in the progenitor disks, $V_{c}$ the circular velocity of the dark matter halo and $f_{b h}$ a free parameter. This scaling provides a good fit to the observed relation between the mass of the super-massive black hole and the velocity dispersion of a galaxy ${ }^{26}$. Following Croton et al. ${ }^{18}$ we here assume the velocity dispersion of a galaxy to be identical to the circular velocity of the dark halo the galaxy is embedded in. This however is not strictly true as e.g. in the case of an isothermal sphere $V_{c} / \sigma=\sqrt{2}$. Several authors have investigated the correlation between $V_{c}$ and $\sigma^{27,28}$ and found different correlations. We here note that using a different scaling between $V_{c}$ and $\sigma$ in our models still produces the same $M_{\bullet}-\sigma$-relation if we adjust the free parameter $f_{b h}$ accordingly. 
Feedback from Super-Massive-Black Holes We here introduce a new prescription for the modelling of feedback from super-massive black holes, which is based on the results presented in this paper. According to our analyses a critical black hole mass exists for a given galaxy velocity dispersion $\sigma$, at which feedback is so strong that it reheats all the available cold gas and hence prevents further star formation. This critical black hole mass is derived from our empirical fit to the residual star formation fraction found in our data as

$$
M_{\bullet, c}=1.26 \times 10^{8}\left(\frac{\sigma}{200}\right)^{3.65}
$$

In galaxies with black holes more massive than the corresponding critical black hole mass we stop any cooling of gas and star formation. We callibrate this relation to give the right fraction of RSF galaxies as seen in Figure 1.

Free Parameters We normalise our two model parameters for the star formation efficiency $\alpha$ and supernova feedback efficiency $\eta$ by matching the $I$-band Tully-Fisher relation of Giovanelli et al. ${ }^{29}$ and requiring that spiral central galaxies of halos with circular velocity $V_{C}=220 \mathrm{~km} / \mathrm{s}$ have on average $10^{11} \mathrm{M}_{\odot}$ of stars and $10^{9} \mathrm{M}_{\odot}$ of cold gas ${ }^{30}$. The third free parameter $f_{b h}$, which regulates the black hole growth is set to match the observed $M_{\bullet}-\sigma$-relation and is $f_{b h}=0.02$.

\section{The Virgo Cluster Data}

We derive the $N U V-V$ colour- $\sigma$ relation for the Virgo cluster down to much smaller values of $\sigma$ than in our GALEX-SDSS sample by combining the near-UV and optical photometry from Boselli et al. ${ }^{4}$ with velocity dispersion measurements from the GOLDmine database ${ }^{31}$. The purpose of 
looking at the Virgo cluster galaxies is to constrain the low end of the $\sigma$ and so get an idea of the point at which all galaxies are RSF galaxies and thus below critical. Since the optical photometry of the Virgo galaxies in this sample are in V-band, we approximately convert the RSF criterion of $N U V-r=5.4$ to $N U V-V$. We use simple stellar populations of solar metallicity and three ages $(3,6$ and $9 \mathrm{Gyr}$ ) to derive the $N U V-V \mathrm{RSF}$ criterion; these are $N U V-V=5.16,5.12$ and 5.09 respectively. In all three cases, the point at which no Virgo early-type galaxy is above this cutoff - i.e. all show some signs of young populations - is at $\sigma \sim 80 \mathrm{kms}^{-1}$.

\section{The RSF criterion}

We use $N U V$-r colour as a discriminant between those galaxies which are quiescent and those which show signs of recent star formation. Besides AGN, there is one further effect which can mimic young stars in the near-UV. Extremely old stellar populations can give rise to the UV upturn phenomenon in some early-type galaxies ${ }^{32,33}$. We choose the $N U V-r$ colour of one of the strongest UV upturn galaxies NGC $4552^{33}$ to be the boundary between galaxies with no young component on the one hand and those that are so blue they must have some young stars in them. Although still quite limited, theoretical population synthesis models which are supported by empirical data (Lee et al. ${ }^{34}$ ) also suggest that the NUV-r of passively evolving ETGs could not be much bluer than 5.4. Using this criterion, we compute the fraction of galaxies that must have experienced some star formation within the last billion years. The ages and mass fractions of these young stellar components are generally $300-500 \mathrm{Myr}$ and $1-3 \%$ by mass $^{35}$. Due to the high sensitivity to dust extinction, these fractions are a lower limit as cold gas and dust have been detected in many early- 
type galaxies ${ }^{36,37} . N U V-r$ probes recent star formation for up to $1 \mathrm{Gyr}$ and so traces different time scales than those of AGN feedback. so there may be a certain amount of time lag between the UV emissions and the processes underlying feedback.

\section{Visual Inspection of Galaxy Morphology}

The process and criteria of the visual inspection are described in detail in Schawinski et al. ${ }^{1}$ In summary, we find early-type galaxy (ETG) candidates from the SDSS database using frac_Dev parameter, which is the weight of the de Vaucouleurs component in the two-component (de Vaucouleurs and exponential disc) fits. We use frac_Dev $>0.95$, which is a highly conservative criterion, hoping to exclude as many spiral interpolers as possible. Despite this, still some spiral bulges remain in our sample. Hence, we remove them via visual inspection on gri bands ${ }^{38}$. Since visual inspections are also subject to errors, we investigat out to what redshift and apparent magnitude visual inspection based on SDSS images is reliable by comparing them with one of the COMBO-17 fields overlapping SDSS. The COMBO-17 image is significantly deeper and has much better seeing with 0.7 " as opposed to 1.4 " which are typical for SDSS. By comparing our classification based on these two different data sets, we concluded that visual inspection of morphology using SDSS was possible to $\mathrm{z} \sim 0.13$ and $\mathrm{R}<17.31$. We settle on more conservative limits of $\mathrm{z}<0.1$ and $\mathrm{r}<16.8$. The presence of late-type interlopers with star-forming disks or spiral arms is therefore not a significant concern and does not give rise to the correlations between $M_{r}, \sigma$ and NUV-r color. A selection of sample images with their velocity dispersions are shown in Figure 4 

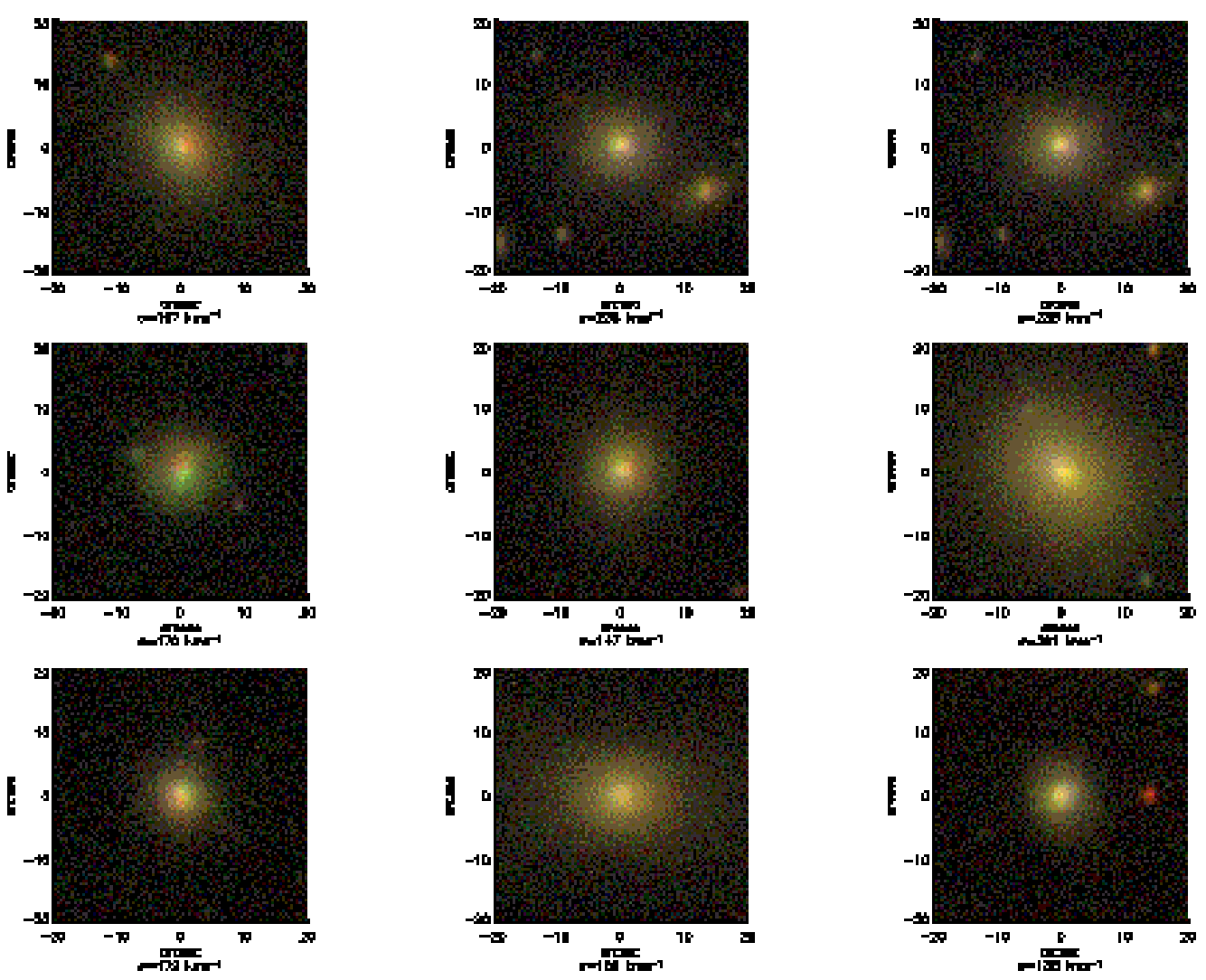

Figure 4: Example galaxies from our SDSS sample. The images are gri $\operatorname{composites}^{38}$ and span a wide range in velocity dispersion. 
1. Schawinski, K. et al. The effect of environment on the UV colour-magnitude relation of earlytype galaxies. ArXiv Astrophysics e-prints (2006). arXiv:astro-ph/0601036.

2. White, S. D. M. \& Frenk, C. S. Galaxy formation through hierarchical clustering. Astrophys. J. 379, 52-79 (1991).

3. Springel, V., White, S. D. M., Tormen, G. \& Kauffmann, G. Populating a cluster of galaxies I. Results at [formmu2]z=0. Mon. Not. R. Astron. Soc. 328, 726-750 (2001).

4. Boselli, A. et al. UV Properties of Early-Type Galaxies in the Virgo Cluster. Astrophys. J. Lett. 629, L29-L32 (2005).

5. Kauffmann, G., Colberg, J. M., Diaferio, A. \& White, S. D. M. Clustering of galaxies in a hierarchical universe - I. Methods and results at z=0. Mon. Not. R. Astron. Soc. 303, 188-206 (1999).

6. Somerville, R. S. \& Kolatt, T. S. How to plant a merger tree. Mon. Not. R. Astron. Soc. 305, $1-14$ (1999).

7. Somerville, R. S., Lemson, G., Kolatt, T. S. \& Dekel, A. Evaluating approximations for halo merging histories. Mon. Not. R. Astron. Soc. 316, 479-490 (2000).

8. Silk, J. On the fragmentation of cosmic gas clouds. I - The formation of galaxies and the first generation of stars. Astrophys. J. 211, 638-648 (1977).

9. White, S. D. M. \& Rees, M. J. Core condensation in heavy halos - A two-stage theory for galaxy formation and clustering. Mon. Not. R. Astron. Soc. 183, 341-358 (1978). 
10. Sutherland, R. S. \& Dopita, M. A. Cooling functions for low-density astrophysical plasmas. Astrophys. J. Supp. 88, 253-327 (1993).

11. Spergel, D. N. et al. First-Year Wilkinson Microwave Anisotropy Probe (WMAP) Observations: Determination of Cosmological Parameters. Astrophys. J. Supp. 148, 175-194 (2003).

12. Navarro, J. F. \& Steinmetz, M. The Effects of a Photoionizing Ultraviolet Background on the Formation of Disk Galaxies. Astrophys. J. 478, 13-+ (1997).

13. Gnedin, N. Y. Effect of Reionization on Structure Formation in the Universe. Astrophys. J. 542, 535-541 (2000).

14. Somerville, R. S. Can Photoionization Squelching Resolve the Substructure Crisis? Astrophys. J. Lett. 572, L23-L26 (2002).

15. Kogut, A. et al. First-Year Wilkinson Microwave Anisotropy Probe (WMAP) Observations: Temperature-Polarization Correlation. Astrophys. J. Supp. 148, 161-173 (2003).

16. Kennicutt, R. C. The Global Schmidt Law in Star-forming Galaxies. Astrophys. J. 498, 541-+ (1998).

17. Scalo, J. M. The stellar initial mass function. Fundamentals of Cosmic Physics 11, 1-278 (1986).

18. Toomre, A. \& Toomre, J. Galactic Bridges and Tails. Astrophys. J. 178, 623-666 (1972).

19. Barnes, J. E. \& Hernquist, L. Dynamics of interacting galaxies. Ann. Rev. Astron. Astrophys. 30, 705-742 (1992). 
20. Burkert, A. \& Naab, T. Major Mergers and the Origin of Elliptical Galaxies. LNP Vol. 626: Galaxies and Chaos 626, 327-339 (2003).

21. Barnes, J. E. \& Hernquist, L. E. Fueling starburst galaxies with gas-rich mergers. Astrophys. J. Lett. 370, L65-L68 (1991).

22. Mihos, J. C. \& Hernquist, L. Gasdynamics and Starbursts in Major Mergers. Astrophys. J. 464, 641-+ (1996).

23. Barnes, J. E. Gas Dynamics in Galaxy Mergers. In ASP Conf. Ser. 240: Gas and Galaxy Evolution, 135-+ (2001).

24. Barnes, J. E. Formation of gas discs in merging galaxies. Mon. Not. R. Astron. Soc. 333, 481-494 (2002).

25. Khochfar, S. \& Burkert, A. The Importance of Spheroidal and Mixed Mergers for Early-Type Galaxy Formation. Astrophys. J. Lett. 597, L117-L120 (2003).

26. Kauffmann, G. \& Haehnelt, M. G. The clustering of galaxies around quasars. Mon. Not. R. Astron. Soc. 332, 529-535 (2002).

27. Ferrarese, L. Beyond the Bulge: A Fundamental Relation between Supermassive Black Holes and Dark Matter Halos. Astrophys. J. 578, 90-97 (2002).

28. Pizzella, A. et al. On the Relation between Circular Velocity and Central Velocity Dispersion in High and Low Surface Brightness Galaxies. Astrophys. J. 631, 785-791 (2005).

29. Giovanelli, R. et al. The Tully-Fisher Relation and H 0. Astrophys. J. Lett. 477, L1+ (1997). 
30. Somerville, R. S. \& Primack, J. R. Semi-analytic modelling of galaxy formation: the local Universe. Mon. Not. R. Astron. Soc. 310, 1087-1110 (1999).

31. Gavazzi, G., Boselli, A., Donati, A., Franzetti, P. \& Scodeggio, M. Introducing GOLDMine: A new galaxy database on the WEB. Astron. Astrophys. 400, 451-455 (2003).

32. Code, A. D. \& Welch, G. A. Ultraviolet photometry from the Orbiting Astronomical Observatory. XXVI - Energy distributions of seven early-type galaxies and the central bulge of M31. Astrophys. J. 228, 95-104 (1979).

33. Burstein, D., Bertola, F., Buson, L. M., Faber, S. M. \& Lauer, T. R. The far-ultraviolet spectra of early-type galaxies. Astrophys. J. 328, 440-462 (1988).

34. Lee, Y.-W. et al. The Look-back Time Evolution of Far-Ultraviolet Flux from Elliptical Galaxies: The Fornax Cluster and A2670. Astrophys. J. Lett. 619, L103-L106 (2005).

35. Kaviraj, S. UV-optical colours as probes of early-type galaxy evolution (in prep.).

36. Knapp, G. R., Guhathakurta, P., Kim, D.-W. \& Jura, M. A. Interstellar matter in early-type galaxies. I - IRAS flux densities. Astrophys. J. Suppl. 70, 329-387 (1989).

37. Temi, P., Brighenti, F., Mathews, W. G. \& Bregman, J. D. Cold Dust in Early-Type Galaxies. I. Observations. Astrophys. J. Suppl. 151, 237-269 (2004).

38. Lupton, R. et al. Preparing Red-Green-Blue Images from CCD Data. Publications of the Astronomical Society of the Pacific 116, 133-137 (2004). 